\title{
Decreased connectivity and increased BOLD complexity in the default mode network in individuals with chronic fatigue syndrome
}

Zack Y. Shan ${ }^{1}$, Kevin Finegan ${ }^{2}$, Sandeep Bhuta ${ }^{2}$, Timothy Ireland ${ }^{2}$, Donald R. Staines ${ }^{1}$, Sonya M. Marshall-Gradisnik ${ }^{1}$, Leighton R. Barnden ${ }^{1}$

${ }^{1}$ National Centre for Neuroimmunology and Emerging Diseases, Menzies Health Institute Queensland, Griffith University, Southport, QLD 4222, Australia

${ }^{2}$ Medical Imaging Department, Gold Coast University Hospital, Parklands, QLD 4215, Australia

Zack Y. Shan: z.shan@griffith.edu.au

Kevin Finegan: Kevin.Finegan@health.qld.gov.au

Sandeep Bhuta: sandeepbhuta@gmail.com

Timothy Ireland: Timothy.Ireland@ health.qld.gov.au

Donald R. Staines: d.staines@griffith.edu.au

Sonya M. Marshall-Gradisnik: s.marshall-gradisnik@griffith.edu.au

Leighton R. Barnden: 1.barnden@ griffith.edu.au

Corresponding Author: Zack Shan, Ph. D.; National Centre for Neuroimmunology and Emerging Diseases, Menzies Health Institute Queensland, Griffith University, Southport, QLD 4222, Australia; T: +61 (7) 56780725, F: +61 (7) 56728908; E: z.shan@griffith.edu.au

Running title: Default mode network dysfunction in CFS

Keywords: chronic fatigue syndrome; default mode network; connectivity; complexity; dysfunction 


\section{Abstract}

The chronic fatigue syndrome / myalgic encephalomyelitis (CFS) is a debilitating disease with unknown pathophysiology and no diagnostic test. This study investigated the default mode network (DMN) in order to understand the pathophysiology of CFS and to identify potential biomarkers.

Using functional MRI (fMRI) collected from 72 subjects (45 CFS and 27 controls) with a temporal resolution of $0.798 \mathrm{~s}$, we evaluated the default mode network using static functional connectivity (FC), dynamic functional connectivity (DFC) and DFC complexity, blood oxygenation level dependent (BOLD) activation maps and complexity of activity. General linear model (GLM) univariate analysis was used for inter group comparison to account for age and gender differences. Hierarchical regression analysis was used to test whether fMRI measures could be used to explain variances of health scores.

BOLD signals in the posterior cingulate cortex (PCC), the driving hub in the DMN, were more complex in CFS in both resting state and task $(\mathrm{P}<0.05)$. The FCs between medial prefrontal cortex (mPFC) and both inferior parietal lobules (IPLs) were weaker $(\mathrm{P}<0.05)$ during resting state, while during task mPFC - left IPL and mPFC - PCC were weaker $(\mathrm{P}<0.05)$. The DFCs between the DMN hubs were more complex in CFS $(\mathrm{P}<0.05)$ during task. Each of these differences accounted for $7-11 \%$ variability of health scores.

This study showed that DMN activity is more complex and less coordinated in CFS, suggesting brain network analysis could be potential used as a diagnostic biomarker for CFS. 


\section{Acronyms}

BOLD: blood oxygenation level dependent; CFS: chronic fatigue syndrome; DFC: dynamic functional connectivity; DMN: default mode network; FC: static functional connectivity; fMRI: functional magnetic resonance imaging; GLM: general linear model; IPL: inferior parietal lobule; MCS: mental component summary; ME: myalgic encephalomyelitis; mPFC: medial prefrontal cortex; PCC: posterior cingulate cortex; PCS: physical component summary; rsfMRI: resting state fMRI; SF-36: 36-item Short Form Health Survey; tfMRI: task fMRI; 


\section{Introduction}

Chronic fatigue syndrome / myalgic encephalomyelitis (CFS/ME) is a debilitating illness characterized by persistent or relapsing fatigue for at least 6 consecutive months, and four or more of: post-exertional malaise, impaired memory or concentration, unrefreshing sleep, muscle pain, multi-joint pain without redness or swelling, tender cervical or axillary lymph nodes, sore throat, and headache (Fukuda et al, 1994). Although the pathophysiology of CFS is not established, altered sensory and pain reception, reduced motor speed, defective attention, and abnormalities in cognition and information processing in CFS suggest that the brain plays a key role in CFS (Holgate et al, 2011).

The main symptoms of fatigue and post exertional malaise suggest dysfunction of energy metabolism in CFS. Metabolomics results show that CFS appears to represent a hypometabolic survival state (decreased metabolites) that is triggered by environment stress (Naviaux et al, 2016). Further, metabolic profiling analysis suggests functional impairment of oxidative metabolism is associated with CFS (Fluge et al, 2016). A recent study on metabolomic analysis of plasma samples showed dysfunction of tricarboxylic acid and urea cycles in CFS patients (Yamano et al, 2016). The brain is one of the most metabolically active organs in the body and is responsible for about $20 \%$ of total energy expenditure (Sokoloff, 1960). The default mode network (DMN) tends to be among the most metabolically active regions in healthy resting subjects (Raichle et al, 2001). Therefore, we hypothesize that the DMN will be abnormal in CFS, either contributing to or being affected by the hypometabolic state in CFS.

The DMN is a network of interacting brain regions that show spontaneous and ongoing brain activity (Raichle et al, 2001). The study of DMN is important for understanding individual brain function since the DMN represents one of the primary functional 
networks of the brain and its function constitutes baseline brain function (WhitfieldGabrieli and Nieto-Castanon, 2012). The DMN mediates processing of one's thoughts and feelings (Buckner et al, 2008; Raichle and Snyder, 2007) and its functional connectivity correlates with cognitive performance (Mak et al, 2017; Persson et al, 2014). These aspects of brain function are both affected in CFS (Holgate et al, 2011). This study used both task fMRI (tfMRI) and resting state fMRI (rsfMRI) to evaluate DMN in CFS in terms of activation, signal complexity, static functional connectivity (FC), and dynamic functional connectivity (DFC) to provide insights into CFS pathophysiology and assess the DMN connectivity as a biomarker for CFS.

\section{Materials and Methods}

Subjects: This study was approved by the Human Research Ethics Committees of our institution and the hospital where scanning was performed. Patients and controls were recruited over a 1-year period. Patients who were undergoing any treatment or taking central nervous system (CNS) medication were excluded. Signed informed consent was obtained from all participants. All the CFS patients met the Fukuda (Fukudaet al, 1994) diagnostic criteria. The total number of subjects analysed in this study was 72 , comprised of 45 CFS patients and 27 normal controls (Table 1). All participants completed the 36-item Short Form Health Survey (SF-36) questionnaire (Ware et al, 1995), in which higher scores suggest better health.

MRI acquisition: The MRI data were acquired on a 3T MRI scanner (Skyra, Siemens) while the subject viewed a video screen through goggles. Three dimensional T1weighted anatomical images were acquired using a T1-weighted magnetization prepared rapid gradient-echo sequence (208 slices, repetition time $(\mathrm{TR})=2400 \mathrm{~ms}$, echo time $(\mathrm{TE})=1.81 \mathrm{~ms}$, flip angle $=8^{\circ}$, acquisition matrix $=224 \times 224$, voxel size $1 \mathrm{~mm} \times$ $1 \mathrm{~mm} \times 1 \mathrm{~mm})$. The fMRI data were acquired using a multiband echo-planar imaging 
(EPI) pulse sequence developed at the University of Minnesota (Auerbach et al, 2013) (72 slices, multiband factor $=8, \mathrm{TR}=798 \mathrm{~ms}, \mathrm{TE}=30 \mathrm{~ms}$, flip angle $=40^{\circ}$, acquisition matrix $=106 \times 106$, voxel size $2 \mathrm{~mm} \times 2 \mathrm{~mm} \times 2 \mathrm{~mm})$. Before each fMRI data acquisition, a single band reference EPI volume and two spin echo EPI volumes encoded with opposite phase directions were acquired. A total of 1100 rsfMRI volumes were acquired over 15 minutes, while the subject was awake and viewing a fixed stationery cross. 1100 tfMRI volumes were acquired over 15 minutes while the subject was performing a sequence of Stroop tasks. The rsfMRI was acquired before tfMRI for all individuals.

Stroop task experimental paradigm: The subjects performed a random event-related design colour word variant of the Stroop task during tfMRI acquisition (Leung et al, 2000). The Stroop task was selected because attention and concentration difficulties are frequent complaints of CFS patients (Ray et al, 1993). The participant was instructed to decide whether the colour of the upper word agreed with the meaning of the lower word and press one of two buttons on a handpiece accordingly. The upper word, consisting of either RED, BLUE, YELLOW, or XXXX, was presented in colours of red, blue, or yellow on a black background. The lower word was either RED, BLUE, or YELLOW coloured white on a black background (Supplementary Fig S1). A total number of 110 trials were randomly distributed over a session of 15 minutes. Among them, $40 \%$ of the trials were incongruent (e.g. the word RED written in blue), $30 \%$ congruent (e.g. BLUE written in blue), and 30\% neutral (e.g. XXXX written in yellow). The Stroop paradigm was encoded using Cogent (The Laboratory of Neurobiology, www.vislab.ucl.ac.uk). The time of stimulus-ON, the response time (RT), and the accuracy were recorded for each subject. The Stroop effect was calculated as the 
average RT of incongruent trials minus the average RT of congruent trials normalized to the average RT of all trials.

MRI pre-processing: Both rsfMRI and tfMRI data were pre-processed as follows. (1) The first five of 1100 fMRI EPI volumes were discarded to ensure that tissue magnetization had reached steady state. (2) Motion correction was applied by registering 1095 EPI volumes to the single band reference image acquired immediately before them using MCFLIRT (Jenkinson et al, 2002) implemented in FSL (FMRIB's Software Library, www.fmrib.ox.ac.uk/fsl). (3) A distortion correction was applied using the distortion field calculated from the two oppositely phase-encoded spin echo EPI volumes using the toolbox "topup" (Andersson et al, 2003) implemented in FSL. (4) The distortion corrected fMRI volumes were coregistered to the corresponding T1 3D anatomic image and then spatially normalized to the Montreal Neurological Institute (MNI) space average brain T1 template (Ashburner and Friston, 1999) using SPM12 (Wellcome Trust Centre for Neuroimaging, London, UK). (5) Normalized volumes were smoothed with a $4 \times 4 \times 4 \mathrm{~mm}^{3}$ full width at half maximum Gaussian kernel using SPM12.

Neural correlates of Stroop task: The tfMRI data were analysed using the two level GLM approach implemented in SPM12. At the subject level, a canonical hemodynamic response function (HRF) with time and dispersion derivatives was used as the basis function. The activation map associated with each type of trial and the difference between task and rest periods were determined using the convolution of the HRF with the neural event as defined by the stimulus-on and subject response times. The congruent, incongruent, neutral, 'Stroop' (incongruent - congruent), task minus rest (positive), and rest minus task (negative) t-contrast maps were entered into a grouplevel analysis. At the group level, we performed random-effect one-sample t-tests to 
identify neural correlates of tasks in each group and two sample t-tests to identify group differences between CFS and NC ( $\alpha<0.05$ with family wise error (FWE) adjustment for multiple comparisons).

Extraction of fMRI time series: Four DMN hubs, mPFC, PCC, L-IPL, and R-IPL were firstly defined from a brain atlas which incorporated cytoarchitectonic, functional and structural connectivity information (Fan et al, 2016). An iterative exclusion at the voxel level based on self-coherence was performed for each DMN hub in each subject as follows: (1) The fMRI time series for each DMN hub was extracted by averaging the signal intensity in the voxels within the region at each time point. (2) For each voxel within a hub, the correlation between the voxel time series and the hub time series was calculated. (3) The voxels that were not significantly correlated $(\mathrm{P}>0.05)$ with the hub average were excluded, then the hub time series was calculated again. (4) Steps (1) to (3) were repeated until no more voxels were excluded. The fMRI time series for each DMN hub was then extracted and smoothed with a high pass filter of $128 \mathrm{~s}$ to remove baseline drifting.

Temporal complexity (sample entropy): The sample entropy (SampEn), a modification of approximate entropy (Pincus, 2006), was used for measurement of system irregularity for time series (Richman and Moorman, 2000). Given a full time series of data with length $N$, the SampEn was calculated as the negative natural logarithm of the probability that segments of data points with length $\mathrm{m}, X_{m}(i)=$ $\left\{x_{i}, x_{i+1}, x_{i+2}, \ldots, x_{i+m-1}\right\}$, that are similar within a tolerance $r$, remain similar when the segments were extended to include the next data point:

$$
\text { SampEn }=-\ln \frac{C_{m+1}}{C_{m}}
$$


where $C_{m}$ is the count of data segments with length $m$ having the Chebyshev distance $d$ smaller than the tolerance $r$. For a segment $X_{m}(i)$, the Chebyshev distance $d$ was defined as:

$$
d\left[X_{m}(i), X_{m}(j)\right](i \neq j)=\underbrace{\max }_{i}\left(\left|x_{i}-x_{j}\right|\right) .
$$

In this study, the length $\mathrm{m}$ was set to 2 and the tolerance $r$ was set to 0.2 times of standard deviation of the full time series.

\section{Static functional connectivity (FC) and dynamic functional connectivity (DFC):}

The FC between any pair of DMN bubs was calculated as the Pearson correlation coefficient between the extracted time series of the DMN pair.

The DFC between each pair of DMN hubs was calculated using a tapered sliding window approach. Tapering reduces spurious physiological noise and sensitivity to outliers (Pozzi et al, 2012; Zalesky et al, 2014). In this approach, for a sliding window with length of $N$, the weighted correlation $\rho_{t}^{i, j}$ between hub $i$ and hub $j$ at time $t \geq N$ was computed as:

$$
\begin{gathered}
\rho_{t}^{i, j}=\sigma_{t}^{i, j} /\left(\sigma_{t}^{i} \sigma_{t}^{j}\right), \text { where } \\
\sigma_{t}^{i}=\sqrt{\sum_{\tau=1}^{N} w_{\tau}\left(x_{t-N+\tau}^{i}-\left\langle x_{t}^{i}\right\rangle\right)} \text {, and } \\
\sigma_{t}^{i, j}=\sum_{\tau=1}^{N} w_{\tau}\left(x_{t-N+\tau}^{i}-\left\langle x_{t}^{i}\right\rangle\right)\left(x_{t-N+\tau}^{j}-\left\langle x_{t}^{i}\right\rangle\right) .
\end{gathered}
$$

The weighted mean $\left\langle x_{t}^{i}\right\rangle$ and the weighting vector $w_{\tau}$ were defined as:

$$
\left\langle x_{t}^{i}\right\rangle=\sum_{\tau=1}^{N} w_{\tau} x_{t-N+\tau}^{i}
$$




$$
w_{\tau}=\left[\left(1-e^{-1 / \theta}\right) /\left(1-e^{-N / \theta}\right)\right] e^{(\tau-N) / \theta}, \tau=1, \ldots, N, \theta>0
$$

In this study, the window length $N$ and the exponent $\theta$ were set to 60 s and 20 s respectively. The DFCs for each DMN hub from 60s to 900s were characterised by the SampEn, the peak amplitude of the fast Fourier transformed (FFT) connectivity spectrum, and the frequency of the peak amplitude in the FFT spectrum.

Statistical analysis: Statistical analysis of the clinical variables and connectivities was performed using SPSS22 (IBM, New York). The Fisher Z-transformation was applied to the FCs (correlation coefficients) before statistical analyses. The significance of difference $(\mathrm{P}<0.05)$ between groups was determined using general linear model $(\mathrm{GLM})$ univariate analysis to account for age and gender differences. The Pearson correlation was used to determine correlation (two-tailed P < 0.05) between MRI measures (DMN BOLD signal SampEns, FCs, and DFC SampEns) and disease severities across all subjects. The MRI measures that were significantly correlated with disease severities were further analysed using a hierarchical regression analysis to determine if MRI measure contributes to variances in health scores across all subjects. In each hierarchical regression, the PCS and MCS were entered as dependent variables; the age and gender were entered as first block independent variables with the forced entry method; each category of the MRI measure (BOLD SampEn, FC, or DFC SampEn) was entered as second block independent variable with the stepwise entry method.

\section{Results}

Demographics and behaviour findings The demographic profile of the 72 subjects is summarized in Table 1. The 45 CFS subjects met the Fukuda CFS criteria (Fukuda et al, 1994). We did not discriminate on the basis of gender during subject recruitment. Therefore, there are more females than males due to the gender difference in CFS 
incidence (Reyes et al, 2003). The NCs were recruited to match the age and female-tomale ratio in the CFS group. The physical component summary (PCS) and mental component summary (MCS) from the SF-36 survey (Ware et al, 1995) in CFS were significantly lower than those in NCs. In the Stroop task the CFS patients scored a slightly lower accuracy and showed less Stroop effect than NCs, but the difference was not significant. However, the CFS patients required significantly longer time than NCs to perform the task (Table 1).

\section{Increased BOLD activation and decreased inhibition in CFS during the Stroop}

task The group-level positive and negative BOLD responses associated with the Stroop task were mapped in Montreal Neurological Institute (MNI) space (Fig. 1). The separate group level one sample t-tests showed that CFS patients have larger regions with increased blood flow and smaller regions with decreased blood flow when engaging in the Stroop task than NCs. However, the two sample t-test comparing CFS patients and NCs was not significant.

PCC BOLD signal fluctuations are more complex in CFS The SampEn of the BOLD signal variation in the PCC was significantly higher in both tfMRI and rsfMRI in CFS patients (1.8 and 1.75) than those in NCs (1.7 and 1.65) (Table 2). The SampEns in the other DMN hubs were not significantly different, so the difference in PCC SampEn could not be attributed to global cerebral blood flow differences. SampEn measures the complexity and disorder of physiological dynamics (Richman and Moorman, 2000). Therefore, this result showed that BOLD signal fluctuations in the PCC are more complex and disordered in CFS patients than NCs. Furthermore, the complexity of rsfMRI BOLD signal in the PCC was negatively correlated $(r=-0.26)$ with SF-36 PCS scores (Supplementary Table S1), that is, poorer physical well-being is associated with increased PCC BOLD complexity. 
Weaker FC among DMN hubs in CFS In the resting state FCs were significantly weaker in CFS patients for mPFC - L-IPL $(P=0.007)$ and mPFC - R-IPL $(P=0.04)$ (Fig 2). During the task the FC for mPFC - PCC was significantly reduced $(P=0.04)$ relative to resting state in CFS patients, while NCs maintained a similar connection strength. Thus, during the task mPFC - PCC connectivity in CFS was weaker than in NCs $(P=0.03)($ Fig 2$)$. Under task conditions the mPFC - R-IPL connectivity in CFS was no longer significantly different to NCs primarily due to a decreased connectivity in the NCs in task relative to rest. The connectivities between mPFC and other DMN hubs (PCC, L- and R-IPL) were significantly correlated with age $(r=-0.25 \sim-0.3)$, with PCS health score $(r=0.21 \sim 0.31)$, and with MCS health score $(r=0.21 \sim 0.29)$ across all subjects (Supplementary Table S2). The hierarchical regression analysis showed that resting $\mathrm{mPFC}-\mathrm{PCC}$ connectivity accounted for $7 \%$ and $8 \%$ variability in the SF-36 PCS and MCS scores (Supplementary Table S3 and S4). However, the hierarchical regression analysis of tfMRI FCs to account for SF-36 PCS or MCS scores retained no explanatory variable.

More complex DFC among DMN hubs in CFS We investigated the connectivity in 60s periods over 15 minutes between the four DMN hubs (Supplementary Fig S3 and S4). Overall, the CFS group showed more irregular DMN connectivity dynamics than NCs (Table 3) in both resting and task states. During the resting period, only the L-IPL - R-IPL connectivity dynamics in CFS patients were significantly more complex than in NCs. When engaging in the cognitive task, however, the mPFC - L-IPL, mPFC PCC, L-IPL - PCC, and R-IPL - PCC connectivity dynamics were all significantly more complex in CFS patients than in NCs. The complexity of resting state timeresolved connectivity for L-IPL - R-IPL and L-IPL - PCC was significantly correlated with PCS $(r=-0.32)$ and MCS $(r=-0.3)$ SF-36 health scores across all subjects, 
respectively (Supplementary Table S5). During task, the complexity of connectivity dynamics of mPFC - PCC was significantly correlated with both PCS $(r=-0.32)$ and MCS $(r=-0.32)$ and the L-IPL - PCC connectivity complexity was significantly correlated with the PCS $(r=-0.28)$ SF-36 health score across all subjects. The hierarchical regression analysis showed that the complexity of time-resolved resting LIPL - R-IPL connectivity accounts for $8 \%$ variability of SF-36 PCS health scores (Supplementary Table S6). The complexity of time-resolved task mPFC - PCC connectivity accounts for 7\% and 11\% variability of SF-36 PCS and MCS health scores (Supplementary Table S7 and S8).

There was no significant difference between CFS and NC groups in maximum amplitude or frequency of maximum amplitude in the power spectrum of time-resolved connectivity for any pair of hubs either during task or resting state.

\section{Discussion}

The purpose of this study was to investigate DMN function in CFS. This is the first study to report complexity measurements of BOLD activity and time-resolved connectivity in CFS. We found that 1) the BOLD activity time series in the PCC was more complex in CFS than NCs in both resting and task states; 2) in the resting state, the time averaged $\mathrm{mPFC}-\mathrm{L}-\mathrm{IPL}$ and $\mathrm{mPFC}-\mathrm{R}$-IPL connectivities were weaker in CFS patients than in NCs, and the time-resolved L-IPL - R-IPL connectivity was more complex in CFS patients than NCs; 3) when engaging in the task, the mPFC - PCC connectivity in CFS patients was weaker than in NCs and the time-resolved connectivity dynamics in the DMN were extensively (mPFC - L-IPL, mPFC - PCC, L-IPL - PCC, and R-IPL - PCC) more complex. 
The DMN is known for its spontaneous and ongoing activity in the resting state without involvement in a task. It constitutes a neurological basis for the self, thinking about others, and remembering the past and thinking about the future (Andrews-Hanna, 2012) and mind wandering (Mason et al, 2007). Therefore, the DMN plays a key role in brain function. Not surprisingly, decreased DMN connectivity has been reported in Alzheimer's disease (Buckner et al, 2008) and sleep deprivation (Basner et al, 2013), both of which demonstrate overlapping clinical features with CFS/ME (attention and memory difficulties). More specifically, our finding of decreased mPFC - IPL connectivity in CFS parallels observations in Alzheimer's disease and sleep deprivation (Basner et al, 2013; Buckner et al, 2008). However, we did not detect a significant difference in $\mathrm{mPFC}$ - PCC connectivity during resting state. The higher complexity in L-IPL - R-IPL connectivity dynamics in CFS may explain the clinical presentation of difficulty in spatial sensing and coordination in CFS.

A case can be made that the PCC is the primary and driving hub for spontaneous activity in the DMN. Firstly, in patients in minimally conscious and vegetative states, their level of consciousness depends on the excitation and connectivity of the PCC (Crone et al, 2015). This suggested that neuronal oscillations in the PCC drive DMN function. Secondly, in the primitive and incomplete DMN in infants aged two weeks, the PCC is the only consistently observed DMN component (Gao et al, 2009). Our novel finding of higher BOLD signal irregularity in the PCC in CFS provides a possible origin for the weaker DMN connectivity observed here in CFS. The high irregularity of PCC BOLD activity (Table 2) could also result in the weaker connectivity between mPFC and PCC in task (Fig. 2). Disrupted PCC activity rhythms may also result in the more complex DFC in the DMN in CFS patients during the task, which here accounted for 7 $-11 \%$ of the disease severity. The higher PCC BOLD SampEns and the associated 
weaker DMN FC are consistent with the reported lower global efficiency (average inverse shortest path length in the network and a measure of information exchange efficiency) in CFS patients (Kim et al. 2015). In healthy subjects, PCC is involved in the neural substrates related to self-evaluation of physical fatigue (Ishii et al. 2014). Our novel finding of higher BOLD SampEns in PCC warrant further investigation of neural processing in the PCC in CFS.

In the resting state the CFS patients had a similar temporal variation in DMN connectivity to NCs (Table 3). However, the complexities of connectivity dynamics in CFS were significantly increased during the task while NCs maintained the same temporal variation for both resting state and task. Thus, DMN synchronization in CFS was weaker than $\mathrm{NC}$ during the task, which more generally may be expected to increase the energy consumption required to perform tasks. Our observation of increased BOLD activation in CFS not balanced by increased BOLD inhibition elsewhere in the brain, together with similar observations in previous reports (Lange et al, 2005; Mizuno et al, 2015), provide support for this notion. It appears that CFS patients demand more BOLD activation to compensate for the more complex and unsynchronized brain activity/connectivity in and between different components of their central nervous system. Increased energy consumption for both baseline functions and tasks would exacerbate, if not cause, fatigue in CFS. These factors may also contribute to the differences in systemic autonomic control reported for the PCC in CFS (Barnden et al, 2016).

This study had two limitations. First, there were fewer NCs than CFS patients due to the difficulty of recruiting age-matched normal subjects. Recently several fMRI databases have become publically available. However, this study investigated the DMN both during the resting state and during a Stroop task and Stroop task fMRI data is not 
yet available. Second, this CFS study only investigated the DMN. Whole brain connectivity was not investigated. Although we established that the DMN in CFS is compromised, altered DMN connectivity has also been observed in other diseases. Therefore, evaluation of the potential of the brain connectome as a specific biomarker for CFS, including its sensitivity, robustness, and reproducibility, must await connectivity studies of its other networks. Future studies will address these two limitations.

In conclusion, the DMN in CFS/ME is abnormal with more irregular activity, weaker connectivity, and more complex connectivity dynamics. In particular, the PCC activity in CFS/ME is more irregular and complex. Further, weaker connectivity among DMN hubs was observed and connection dynamics were more complex especially during the cognitive task. We argue that deficits in DMN could be energy expensive and may contribute to or cause the fatigue, cognitive symptoms and post exertional malaise of CFS. 
We thank the patients and healthy controls who donated their time and effort to participate in this study. This study was supported by the Stafford Fox Medical Research Foundation, the Judith Jane Mason Foundation, Mr Douglas Stutt, and BlakeBeckett Foundation. The financial support does not affect any aspect of the study.

\section{Author Disclosure Statement}

All the authors have no competing financial interests exist. 


\section{References}

Andersson JL, Skare S, Ashburner J. 2003. How to correct susceptibility distortions in spin-echo echo-planar images: application to diffusion tensor imaging. NeuroImage 20:870-888.

Andrews-Hanna JR. 2012. The brain's default network and its adaptive role in internal mentation. Neuroscientist 18:251-270.

Ashburner J, Friston KJ. 1999. Nonlinear spatial normalization using basis functions. Human Brain Mapping 7:254-266.

Auerbach EJ, Xu J, Yacoub E, Moeller S, Ugurbil K. 2013. Multiband accelerated spinecho echo planar imaging with reduced peak RF power using time-shifted RF pulses. Magn Reson Med 69:1261-1267.

Barnden LR, Kwiatek R, Crouch B, Burnet R, Del Fante P. 2016. Autonomic correlations with MRI are abnormal in the brainstem vasomotor centre in Chronic Fatigue Syndrome. NeuroImage: Clinical 11:530-537.

Basner M, Rao H, Goel N, Dinges DF. 2013. Sleep deprivation and neurobehavioral dynamics. Curr Opin Neurobiol 23:854-863.

Buckner RL, Andrews-Hanna JR, Schacter DL. 2008. The brain's default network: anatomy, function, and relevance to disease. Ann N Y Acad Sci 1124:1-38.

Crone JS, Schurz M, Holler Y, Bergmann J, Monti M, Schmid E et al. 2015. Impaired consciousness is linked to changes in effective connectivity of the posterior cingulate cortex within the default mode network. NeuroImage 110:101-109.

Fan L, Li H, Zhuo J, Zhang Y, Wang J, Chen L et al. 2016. The Human Brainnetome Atlas: A New Brain Atlas Based on Connectional Architecture. Cerebral Cortex 26:3508-3526.

Fluge O, Mella O, Bruland O, Risa K, Dyrstad SE, Alme K et al. 2016. Metabolic profiling indicates impaired pyruvate dehydrogenase function in myalgic encephalopathy/chronic fatigue syndrome. JCI Insight 1:e89376.

Fukuda K, Straus SE, Hickie I, Sharpe MC, Dobbins JG, Komaroff A. 1994. The chronic fatigue syndrome: a comprehensive approach to its definition and study. Ann Intern Med 121:953-959.

Gao W, Zhu H, Giovanello KS, Smith JK, Shen D, Gilmore JH, Lin W. 2009. Evidence on the emergence of the brain's default network from 2-week-old to 2-year-old healthy pediatric subjects. Proc Natl Acad Sci U S A 106:6790-6795.

Holgate S, Komaroff A, Mangan D, Wessely S. 2011. Chronic fatigue syndrome: understanding a complex illness. Nat Rev Neurosci 12:539-544. 
Ishii A, Tanaka M, Yamano E, Watanabe Y. 2014. The neural substrates of physical fatigue sensation to evaluate ourselves: a magnetoencephalography study. Neuroscience 261:60-67.

Jenkinson M, Bannister P, Brady M, Smith S. 2002. Improved optimization for the robust and accurate linear registration and motion correction of brain images. NeuroImage 17:825-841.

Kim BH, Namkoong K, Kim JJ, Lee S, Yoon KJ, Choi M, Jung YC. 2015. Altered resting-state functional connectivity in women with chronic fatigue syndrome. Psychiatry Res 234:292-297.

Lange G, Steffener T, Cook D, Bly B, Christodoulou C, Liu W-Cet al. 2005. Objective evidence of cognitive complaints in Chronic Fatigue Syndrome: A BOLD fMRI study of verbal working memory. NeuroImage 26:513- 524.

Leung HC, Skudlarski P, Gatenby JC, Peterson BS, Gore JC. 2000. An event-related functional MRI study of the stroop color word interference task. Cerebral Cortex 10:552-560.

Mak LE, Minuzzi L, MacQueen G, Hall G, Kennedy SH, Milev R. 2017. The Default Mode Network in Healthy Individuals: A Systematic Review and Meta-Analysis. Brain Connect 7:25-33.

Mason MF, Norton MI, Van Horn JD, Wegner DM, Grafton ST, Macrae CN. 2007. Wandering minds: the default network and stimulus-independent thought. Science 315:393-395.

Mizuno K, Tanaka M, Tanabe HC, Joudoi T, Kawatani J, Shigihara Y et al. 2015. Less efficient and costly processes of frontal cortex in childhood chronic fatigue syndrome. NeuroImage: Clinical 9:355-368.

Naviaux RK, Naviaux JC, Li K, Bright AT, Alaynick WA, Wang L et al. 2016. Metabolic features of chronic fatigue syndrome. Proc Natl Acad Sci U S A 113:E54725480 .

Persson J, Pudas S, Nilsson LG, Nyberg L. 2014. Longitudinal assessment of defaultmode brain function in aging. Neurobiol Aging 35:2107-2117.

Pincus SM. 2006. Approximate entropy as a measure of irregularity for psychiatric serial metrics. Bipolar Disord 8:430-440.

Pozzi F, Di Matteo T, Aste T. 2012. Exponential smoothing weighted correlations. Eur Phys J B 85:1-21.

Raichle ME, MacLeod AM, Snyder AZ, Powers WJ, Gusnard DA, Shulman GL. 2001. A default mode of brain function. Proc Natl Acad Sci 98:676-682.

Raichle ME, Snyder AZ. 2007. A default mode of brain function: a brief history of an evolving idea. Neuroimage 37:1083-1090. 
Ray C, Phillips L, Weir WR. 1993. Quality of attention in chronic fatigue syndrome: subjective reports of everyday attention and cognitive difficulty, and performance on tasks of focused attention. Br J Clin Psychol 32 ( Pt 3):357-364.

Reyes M, Nisenbaum R, Hoaglin DC, Unger ER, Emmons C, Randall Bet al. 2003. Prevalence and incidence of chronic fatigue syndrome in Wichita, Kansas. Arch Intern Med 163:1530-1536.

Richman JS, Moorman JR. 2000. Physiological time-series analysis using approximate entropy and sample entropy. Am J Physiol Heart Circ Physiol 278:H2039-2049.

Sokoloff L. 1960 The metabolism of the central nervous system in vivo. In: Field, JM, H W; Hall, V E., editor. Handbook of Physiology-Neurophysiology. Washington, D.C.: American Physiological Society. p 1843-1864.

Ware JE, Jr., Kosinski M, Bayliss MS, McHorney CA, Rogers WH, Raczek A. 1995. Comparison of methods for the scoring and statistical analysis of SF-36 health profile and summary measures: summary of results from the Medical Outcomes Study. Medical Care 33:As264-279.

Whitfield-Gabrieli S, Nieto-Castanon A. 2012. Conn: a functional connectivity toolbox for correlated and anticorrelated brain networks. Brain Connect 2:125-141.

Yamano E, Sugimoto M, Hirayama A, Kume S, Yamato M, Jin G et al. 2016. Index markers of chronic fatigue syndrome with dysfunction of TCA and urea cycles. Sci Rep 6:34990. doi: 10.1038/srep34990.

Zalesky A, Fornito A, Cocchi L, Gollo LL, Breakspear M. 2014. Time-resolved restingstate brain networks. Proc Natl Acad Sci U S A 111:10341-10346. 
Figure Captions

Fig. 1. Group level positive and negative BOLD signal changes associated with the Stroop task. From left to right are shown the left lateral and medial views of the left hemisphere and the medial and right lateral views of the right hemisphere. The group level activation maps show that the CFS patients showed stronger and more extended positive BOLD responses (top 2 rows) and weaker negative BOLD responses (lower 2 rows) than the NCs.

Fig. 2. The time-averaged connectivity between the DMN hubs in NCs (A and C) and in CFS patients (B and D) using rsfMRI (A and B) and tfMRI (C and D). The cyan, purple, orange, and green regions on the image represent the four default mode network (DMN) hubs studied: the medial prefrontal cortex (mPFC), posterior cingulate cortex (PCC), and left (L-) and right (R-) inferior parietal lobule (IPL) respectively. In the resting state, connectivities between the MPFC and L- and R-IPL were lower in CFS patients than in NCs. When engaging in the task, NCs maintained similar connectivities to resting state, except between the mPFC and R-IPL. However, during task the mPFC - PCC connectivity was significantly lowered in CFS compared with NCs and with CFS at rest. 


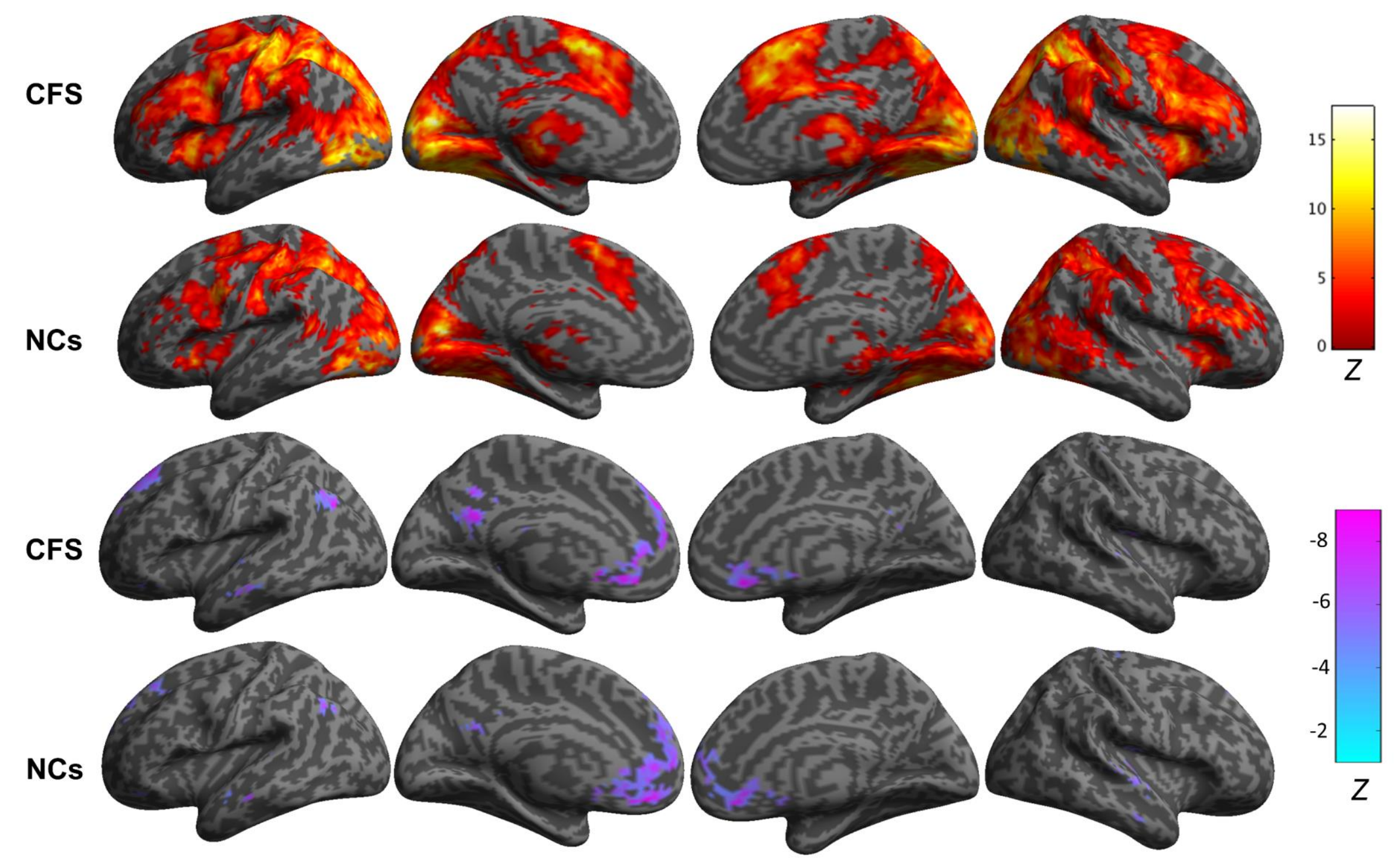




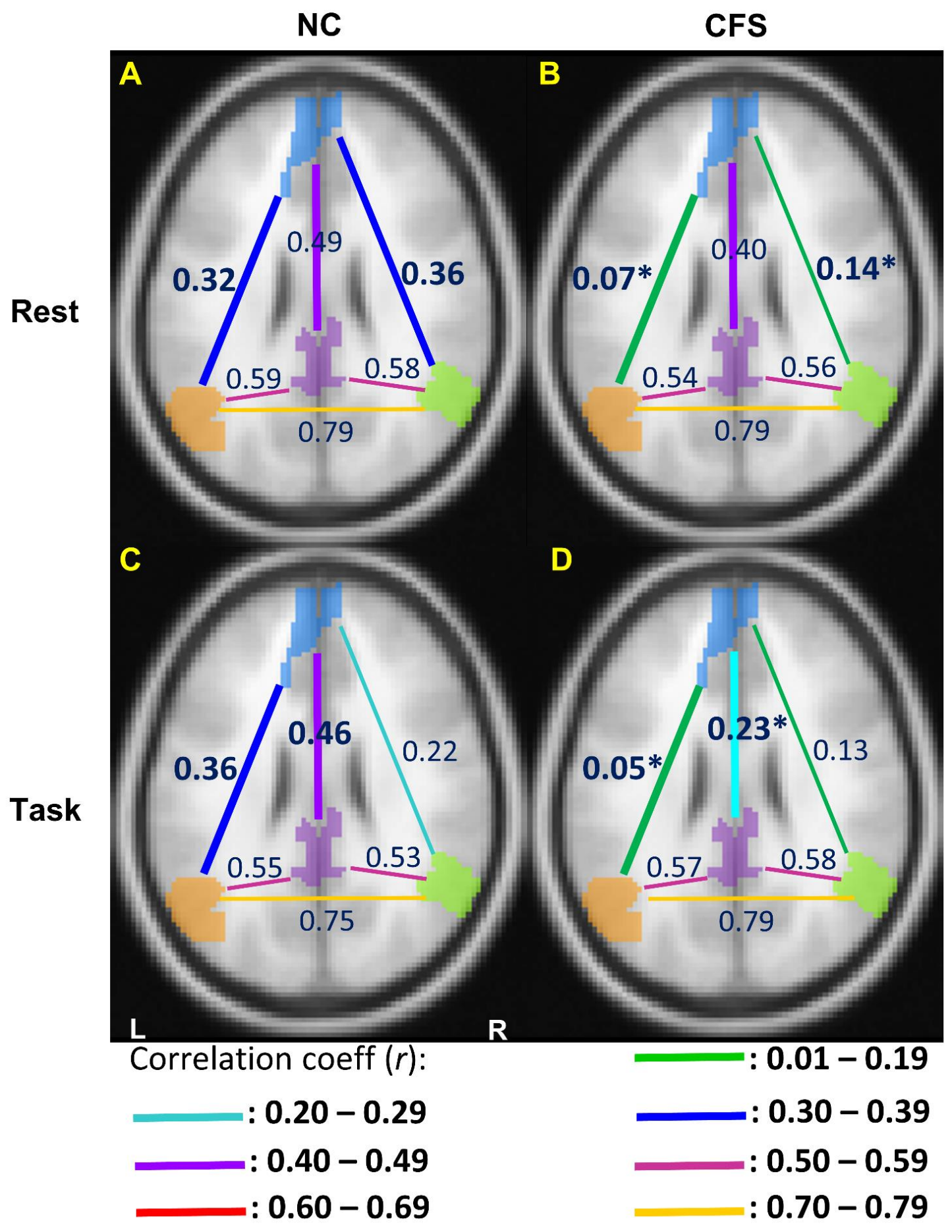




\section{Supplementary Materials}

Decreased connectivity and increased BOLD complexity in the

default mode network in individuals with chronic fatigue syndrome

\section{Table S1}

Pearson correlations between BOLD SampEn and age and health scores across all subjects

\begin{tabular}{lcccc}
\hline & hub & Age & SF-36 PCS & SF-36 MCS \\
\hline \multirow{2}{*}{ tfMRI } & mPFC & $\mathbf{0 . 2 9 *}$ & -0.17 & -0.16 \\
& PCC & $\mathbf{0 . 3 1 * *}$ & -0.14 & -0.07 \\
& L-IPL & $\mathbf{0 . 2 2 *}$ & -0.13 & -0.14 \\
& R-IPL & $\mathbf{0 . 3 2 * *}$ & -0.12 & -0.09 \\
\hline rsfMRI & mPFC & 0.1 & -0.12 & -0.03 \\
& PCC & $\mathbf{0 . 2 5 *}$ & $\mathbf{- 0 . 2 6 *}$ & -0.12 \\
& L-IPL & 0.12 & -0.15 & -0.09 \\
& R-IPL & 0.2 & -0.19 & -0.12 \\
\hline
\end{tabular}

$*: \mathrm{P}<0.05 ; * *: \mathrm{P}<0.01$. 
Table S2

Pearson correlations between connectivity and age and health scores across all subjects

\begin{tabular}{|c|c|c|c|c|}
\hline & Hub pair & Age & SF-36 PCS & SF-36 MCS \\
\hline \multirow{6}{*}{ rsfMRI } & mPFC - L-IPL & $-0.27 *$ & $0.25 *$ & $0.23 *$ \\
\hline & mPFC - R-IPL & $-0.26^{*}$ & $0.21 *$ & $0.21 *$ \\
\hline & $\mathrm{mPFC}-\mathrm{PCC}$ & -0.18 & $0.31 * *$ & $0.29 * *$ \\
\hline & L-IPL - R-IPL & -0.04 & 0.1 & 0.08 \\
\hline & L-IPL - PCC & -0.03 & 0.17 & -0.09 \\
\hline & R-IPL - PCC & -0.11 & 0.18 & 0.14 \\
\hline \multirow{6}{*}{ tfMRI } & mPFC - L-IPL & $-0.29 * *$ & $0.27 *$ & $0.25 *$ \\
\hline & mPFC - R-IPL & $-0.25^{*}$ & $0.27 *$ & $0.26 *$ \\
\hline & $\mathrm{mPFC}-\mathrm{PCC}$ & $-0.3 * *$ & $0.28 *$ & $0.23 *$ \\
\hline & L-IPL - R-IPL & -0.02 & 0.25 & 0.21 \\
\hline & L-IPL - PCC & -0.02 & 0.2 & 0.13 \\
\hline & R-IPL - PCC & -0.03 & 0.21 & 0.2 \\
\hline
\end{tabular}

$*: \mathrm{P}<0.05 ; * *: \mathrm{P}<0.01$. 


\section{Table S3}

Hierarchical regression of resting connectivities predicting PCS health score across all

\begin{tabular}{|c|c|c|c|c|}
\hline & & $b$ & $S E b$ & $\bar{\beta}$ \\
\hline \multirow[t]{3}{*}{ Step 1} & Constant & 77.19 & 16.8 & \\
\hline & Age & -0.7 & 0.36 & -0.24 \\
\hline & Gender & 9.88 & 8.94 & 0.14 \\
\hline \multirow[t]{4}{*}{ Step 2} & Constant & 59.59 & 18.09 & \\
\hline & Age & -0.6 & 0.35 & -0.21 \\
\hline & Gender & 8.34 & 8.67 & 0.12 \\
\hline & $Z_{m P F C-P C C}$ & 30.38 & 13.74 & $0.27^{*}$ \\
\hline
\end{tabular}

${ }^{\S} \mathrm{R}^{2}=0.08$ for step $1 \overline{1, \Delta \mathrm{R}^{2}=0.07 \text {, the significance of } \mathrm{F} \text { change }}<0.05 ; *$ : $\mathrm{P}<0.05$. Hierarchical regression analysis was performed to determine if resting connectivities of mPFC - L-IPL, mPFC - R-IPL, and mPFC - PCC, measured as the Fisher ztransformed correlation coefficient, can account for the variance of the physical component summary (PCS) SF-36 health score. The age and gender were entered in the step 1 hierarchical regression with force entry method. The resting connectivities of mPFC - L-IPL, mPFC - R-IPL, and mPFC - PCC were entered in the step 2 with stepwise entry method. The unstandardized coefficients $(b)$ and their standard error (SE b) and standardized coefficient $(\beta)$ are shown. $\Delta \mathrm{R}^{2}=0.08$ suggested that $8 \%$ of the PCS score variability was accounted for by the resting $\mathrm{mPFC}-\mathrm{PCC}$ connectivity. 


\section{Table S4}

Hierarchical regression of resting connectivities predicting MCS health score across

$$
\text { all subjects }{ }^{\S}
$$

\begin{tabular}{ccccc}
\hline & & $b$ & SE $b$ & $\beta$ \\
\hline Step 1 & Constant & 51.67 & 15.39 & \\
& Age & -0.07 & 0.33 & -0.03 \\
& Gender & 8.16 & 8.19 & 0.13 \\
Step 2 & Constant & 35.87 & 16.61 & \\
& Age & 0.2 & 0.32 & 0.01 \\
& Gender & 6.78 & 7.96 & 0.11 \\
& $Z_{\text {mPFC-PCC }}$ & 27.27 & 12.61 & $\mathbf{0 . 2 8}$
\end{tabular}

$\S: \mathrm{R}^{2}=0.02$ for step $\overline{1, \Delta \mathrm{R}^{2}=0.08 \text {, the significance of } \mathrm{F} \text { change }}<0.05 ; *$ : $\mathrm{P}<0.05$. Hierarchical regression analysis was performed to determine resting connectivities of mPFC - L-IPL, mPFC - R-IPL, and mPFC - PCC, measured as the Fisher ztransformed correlation coefficient, can account for the variance of the mental component summary (MCS) SF-36 health score. The age and gender were entered in the step 1 hierarchical regression with force entry method. The resting connectivities of mPFC - L-IPL, mPFC - R-IPL, and mPFC - PCC were entered in the step 2 with stepwise entry method. The unstandardized coefficients (b) and their standard error (SE b) and standardized coefficient $(\beta)$ are shown. $\Delta \mathrm{R} 2=0.08$ suggested that $8 \%$ of the MCS score variability was accounted for by the resting mPFC-PCC connectivity. 


\section{Table S5}

Pearson correlations between the complexity of connectivity dynamics and age and health scores across all subjects

\begin{tabular}{|c|c|c|c|c|}
\hline & & Age & SF-36 PCS & SF-36 MCS \\
\hline \multirow{6}{*}{ rsfMRI } & mPFC - L-IPL & 0.2 & -0.02 & -0.12 \\
\hline & mPFC - R-IPL & 0.23 & -0.09 & -0.08 \\
\hline & $\mathrm{mPFC}-\mathrm{PCC}$ & $0.35 * *$ & -0.16 & -0.12 \\
\hline & L-IPL - R-IPL & 0.18 & $-0.32 *$ & 0.08 \\
\hline & L-IPL - PCC & $0.29 *$ & -0.19 & $-0.3 *$ \\
\hline & R-IPL - PCC & $0.29 *$ & -0.2 & -0.13 \\
\hline \multirow{6}{*}{ tfMRI } & mPFC - L-IPL & 0.23 & -0.12 & -0.11 \\
\hline & mPFC - R-IPL & $0.29 *$ & -0.07 & -0.11 \\
\hline & $\mathrm{mPFC}-\mathrm{PCC}$ & $0.34 * *$ & $-0.32 *$ & $-0.32 *$ \\
\hline & L-IPL - R-IPL & 0.25 & -0.15 & -0.06 \\
\hline & L-IPL - PCC & $0.38 * *$ & $-0.28 *$ & -0.12 \\
\hline & R-IPL - PCC & $0.46^{* *}$ & -0.22 & -0.16 \\
\hline
\end{tabular}

*: $\mathrm{P}<0.05 ; * *: \mathrm{P}<0.01$. The bivariate correlations between the sample entropy (SampEn) of time-resolved connectivities in each pair of default mode network (DMN) hubs and the age and physical component summary (PCS) and mental component summary (MCS) in SF-36 health scores. Most of the time-resolved connectivity complexities are positively correlated with age, suggesting that the connectivity dynamics become more irregular with aging. 


\section{Table S6}

Hierarchical regression of the complexity of time-resolved resting state connectivities in predicting PCS health score across all subjects ${ }^{\S}$

\begin{tabular}{lcccc}
\hline & & $b$ & SE $b$ & $\beta$ \\
\hline Step 1 & Constant & 63.46 & 15.75 & \\
& Age & -0.37 & 0.33 & -0.14 \\
& Gender & 10.69 & 9.03 & 0.15 \\
Step 2 & Constant & 82.42 & 17.32 & \\
& Age & -0.23 & 0.32 & -0.09 \\
& Gender & 7.71 & 8.82 & 0.11 \\
& SampEnL-IPL-R-IPL & -76.54 & 33.37 & $\mathbf{- 0 . 2 9 *}$
\end{tabular}

$\S: \mathrm{R}^{2}=0.02$ for the step $1, \Delta \mathrm{R}^{2}=0.08$, the significance of $\mathrm{F}$ change $<0.05 ; *: \mathrm{P}<0.05$. The hierarchical regression analysis was performed to determine if complexity of timeresolved resting state L-IPL - R-IPL connectivity, measured as sample entropy (SampEn) of the dynamic connectivity between the left and right inferior parietal lobule (IPL), can predict the variance of the physical component summary (PCS) SF-36 health score. The age and gender were entered in the step 1 hierarchical regression with force entry method. The complexity of time-resolved resting state connectivities of L-IPL R-IPL was entered in the step 2 with stepwise entry method. The unstandardized coefficients (b) and their standard error (SE b) and standardized coefficient $(\beta)$ are shown. $\Delta \mathrm{R} 2=0.08$ suggested that $8 \%$ of PCS score variability was accounted for by the complexity of time-resolved resting L-IPL - R-IPL connectivity. 


\section{Table S7}

Hierarchical regression of the complexities of time-resolved task connectivities predicting PCS health score across all subjects ${ }^{\S}$

\begin{tabular}{lcccc}
\hline & & $b$ & SE $b$ & $\beta$ \\
\hline Step 1 & Constant & 63.46 & 15.75 & \\
& Age & -0.36 & 0.33 & -0.14 \\
& Gender & 10.69 & 9.03 & 0.15 \\
Step 2 & Constant & 81.59 & 17.38 & \\
& Age & -0.11 & 0.34 & -0.04 \\
& Gender & 8.29 & 8.83 & 0.12 \\
& SampEn & -65.49 & 29.96 & $-\mathbf{0 . 2 9 *}$
\end{tabular}

$\S: \mathrm{R}^{2}=0.04$ for the step $1, \Delta \mathrm{R}^{2}=0.07$, the significance of $\mathrm{F}$ change $<0.05 ; *: \mathrm{P}<0.05$. The hierarchical regression analysis was performed to determine if complexities of time-resolved connectivities of tfMRI mPFC - PCC and L-IPL - R-IPL can predict the variance of the physical component summary (PCS) SF-36 health score. The age and gender were entered in the step 1 hierarchical regression with force entry method. The complexities of time-resolved tfMRI connectivities of tfMRI mPFC - PCC and L-IPL - R-IPL were entered in the step 2 with stepwise entry method. The unstandardized coefficients (b) and their standard error (SE b) and standardized coefficient $(\beta)$ are shown. $\Delta \mathrm{R}^{2}=0.07$ suggested that $7 \%$ of PCS score variability was accounted for by the complexity of time-resolved tfMRI mPFC - PCC connectivity. 
Table S8

Hierarchical regression of the complexities of time-resolved task connectivities predicting MCS health score across all subjects ${ }^{\S}$

\begin{tabular}{lcccc}
\hline & & $b$ & SE $b$ & $\beta$ \\
\hline Step 1 & Constant & 46.14 & 13.99 & \\
& Age & 0.07 & 0.29 & 0.32 \\
& Gender & 8.42 & 8.02 & 0.13 \\
Step 2 & Constant & 66.05 & 15.11 & \\
& Age & 0.36 & 0.3 & 0.16 \\
& Gender & 5.78 & 7.67 & 0.09 \\
& SampEn & -71.92 & 26.03 & $\mathbf{- 0 . 3 6 *}$
\end{tabular}

$\S: \mathrm{R}^{2}=0.02$ for the step $1, \Delta \mathrm{R}^{2}=0.11$, the significance of $\mathrm{F}$ change $<0.05 ; *: \mathrm{P}<0.05$. The hierarchical regression analysis was performed to determine if complexity of timeresolved tfMRI mPFC - PCC connectivity can predict the variance of the mental component summary (MCS) SF-36 health score. The age and gender were entered in the step 1 hierarchical regression with force entry method. The complexity of timeresolved tfMRI $\mathrm{mPFC}$ - PCC connectivity was entered in the step 2 with stepwise entry method. The unstandardized coefficients (b) and their standard error (SE b) and standardized coefficient $(\beta)$ are shown. $\Delta \mathrm{R}^{2}=0.11$ suggested that $11 \%$ of MCS score variability was accounted for by the complexity of time-resolved tfMRI mPFC - PCC connectivity. 
Default mode network dysfunction in CFS

Zack Y. Shan et al. 2017

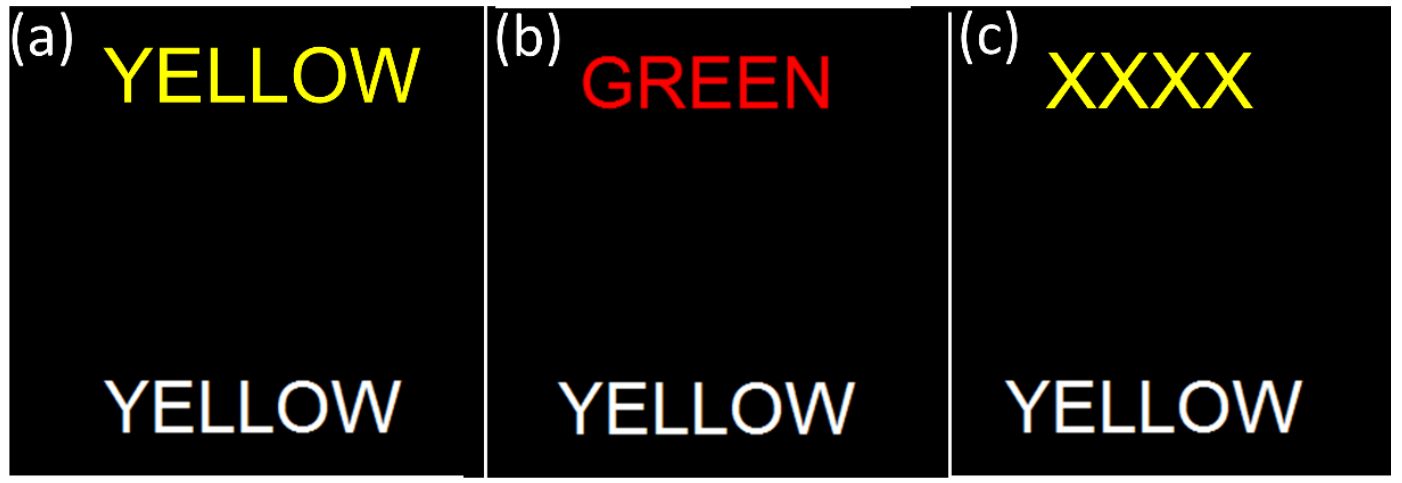

Supplementary Fig 1. Three examples of the colour-word version of the Stroop task. The task is to decide whether the meaning of the lower word corresponds to the colour of the upper word. Examples of (a) congruent, (b) incongruent, and (c) neutral tasks are shown. 


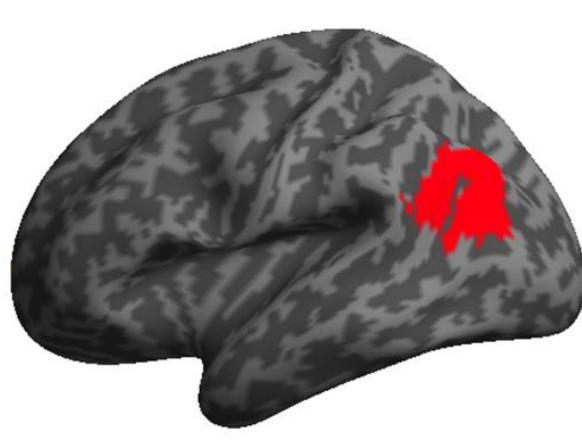

Left lateral

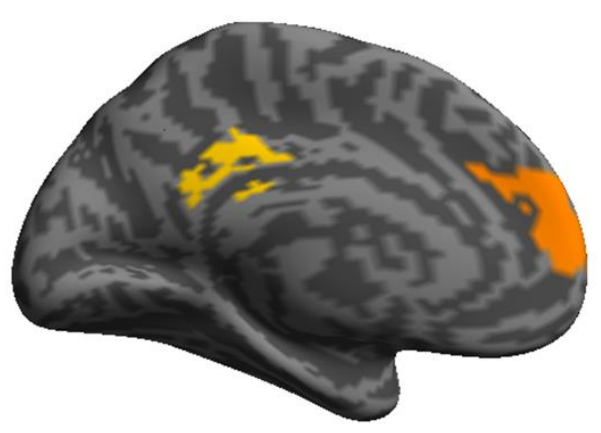

Left medial

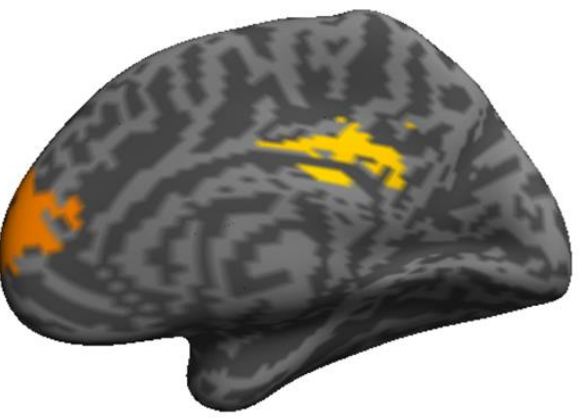

Right medial

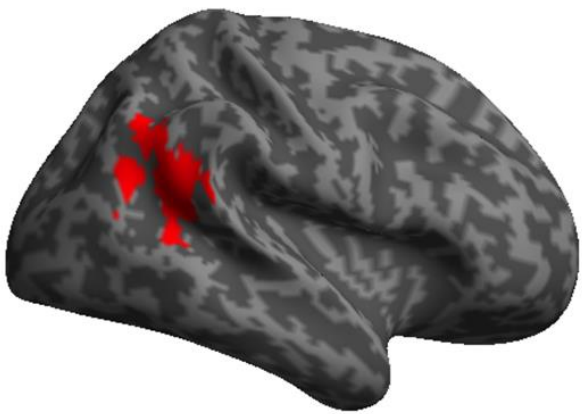

Right lateral

Supplementary Fig. 2. The four default mode network (DMN) hubs used here as defined by the atlas. From the left to the right are the left lateral and medial views of the left hemisphere and the medial and right lateral views of the right hemisphere. Four DMN hubs were investigated in this study: medial prefrontal cortex (orange, cyto-architectonic medial area 10), left- and right-inferior parietal lobule (red, cyto-architectonic rostroventral area 39), and posterior cingulate cortex (yellow, cyto-architectonic dorsal area 23). 
Default mode network dysfunction in CFS
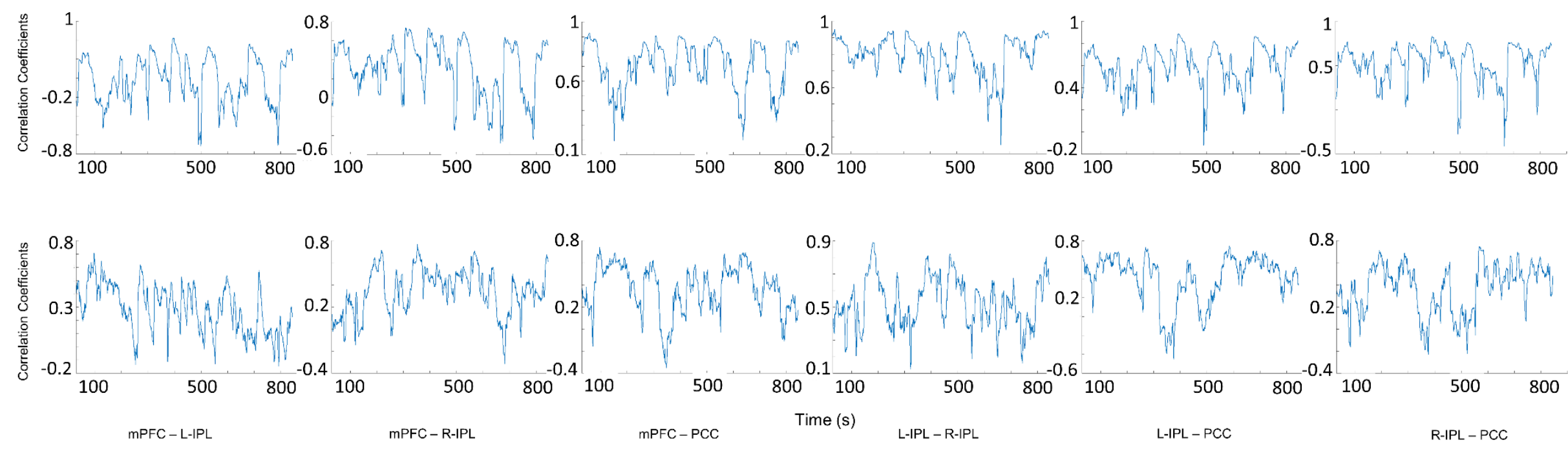

Supplementary Fig. 3. Time resolved connectivity between four default mode network (DMN) hubs calculated using a sliding 60 s window in a 15 minute rsfMRI. The upper row shows dynamic connectivity from a normal control (NC) and the lower row from a patient with chronic fatigue syndrome (CFS). Connectivity dynamics are shown between medial prefrontal cortex (mPFC) and left (L-) inferior parietal lobule (IPL), between $\mathrm{mPFC}$ and right (R-) IPL, between $\mathrm{mPFC}$ and posterior cingulate cortex (PCC), between L-IPL and R-IPL, between L-IPL and PCC, and between R-IPL and PCC. 
Default mode network dysfunction in CFS
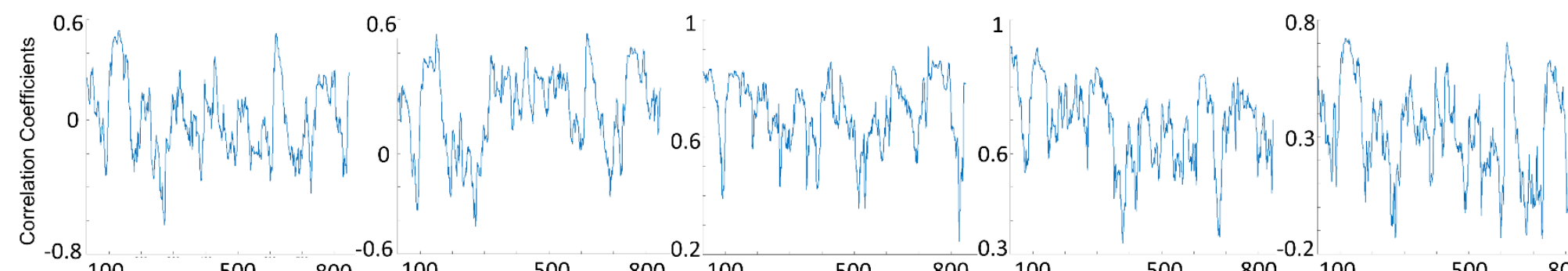

\section{1}

Zack Y. Shan et al. 2017

$500 \quad 800_{0.8}^{-0.2} 100$
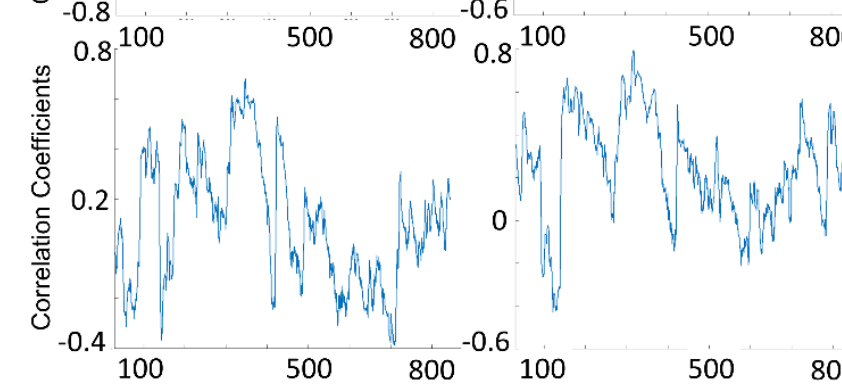

mPFC - L-IPL

mPFC - R-IPL
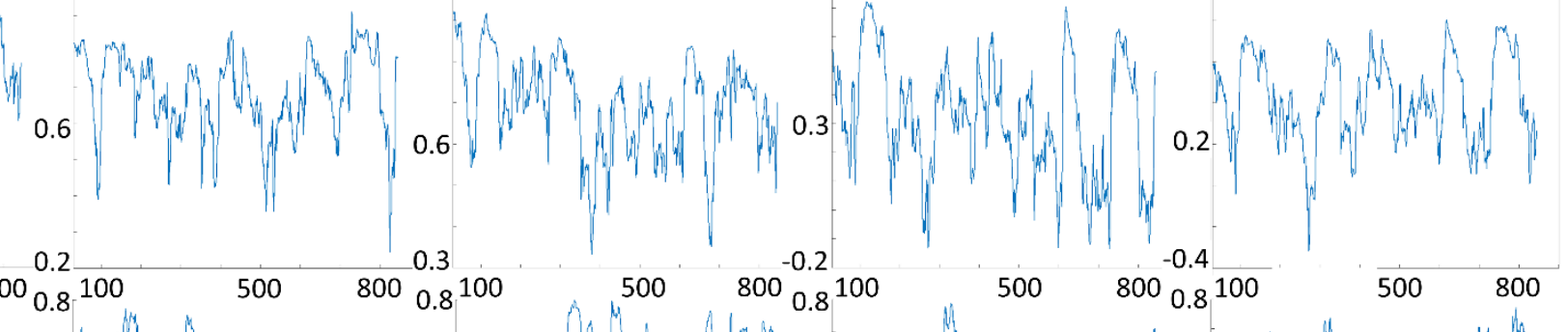

0.2
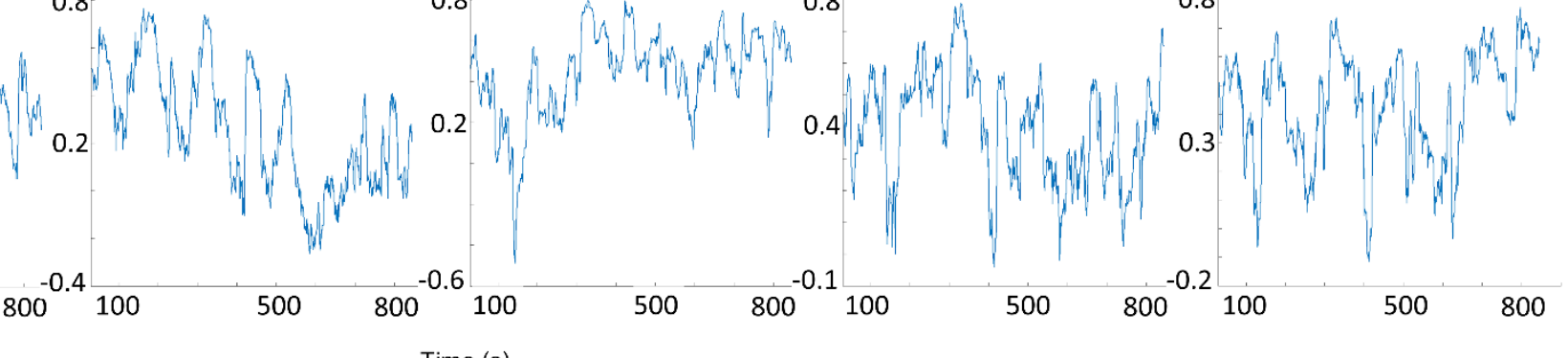

L-IPL - R-IPL

L-IPL - PCC

R-IPL - PCC

Supplementary Fig. 4. Time resolved connectivity between four default mode network (DMN) hubs calculated using a sliding 60s window in a 15 minute tfMRI. The upper row shows dynamic connectivity from a normal control (NC) and the lower row from a patient with chronic fatigue syndrome (CFS). Connectivity dynamics are shown between medial prefrontal cortex (mPFC) and left (L-) inferior parietal lobule (IPL), between mPFC and right (R-) IPL, between mPFC and posterior cingulate cortex (PCC), between L-IPL and R-IPL, between L-IPL and PCC, and between R-IPL and PCC. The connectivities in the CFS patient are more sporadic and irregular than those in the NCs. 